\title{
CHARACTERIZATIONS OF BOUNDED SETS IN SPACES OF ULTRADISTRIBUTIONS
}

\author{
STEVAN PILIPOVIĆ
}

(Communicated by Palle E. T. Jorgensen)

\begin{abstract}
We characterize bounded sets in ultradistributions spaces $\mathscr{D}_{L^{t}}^{\prime\left(M_{p}\right)}$, $t \in[1, \infty], S^{\prime\left\{M_{p}\right\}}$, and $S^{\prime\left(M_{p}\right)}$ and bounded sets and convergent sequences in $\mathscr{D}^{\prime}\left(M_{p}\right)$ and $\mathscr{D}^{\prime}\left\{M_{p}\right\}$ via the convolution by corresponding test functions. The structural theorems for $\mathscr{D}_{L^{t}}^{\prime\left\{M_{p}\right\}}$ and $\widetilde{\mathscr{D}}_{L^{t}}^{\prime\left\{M_{p}\right\}}, t \in[1, \infty]$, are also given.
\end{abstract}

\section{INTRODUCTION}

For the analysis of Beurling and Roumieau spaces of ultradistributions and the background information we refer to [6] and references therein. In this paper we investigate the bounded sets in ultradistribution spaces. The results are analogous to Schwartz's results for distributions.

A characterization of a bounded set in $\mathscr{D}_{L^{i}}^{\prime\left\{M_{p}\right\}}, t \in[1, \infty]$, and representation theorems for the elements of $\mathscr{D}_{L^{t}}^{\prime\left\{M_{p}\right\}}, t \in[1, \infty]$, are given in Lemma 2. In Lemma 3 spaces $\mathscr{D}_{L^{t}}^{\left\{M_{p}\right\}}$ and $\mathscr{D}_{L^{s}}^{\left\{M_{p}\right\}}, s \in[1, \infty)$, and $\dot{\mathscr{B}}\left\{M_{p}\right\}$ and $\dot{\mathscr{\mathscr { B }}}\left\{M_{p}\right\}$ are compared. They are equal in the set-theoretical sense, but we do not know whether they are topologically equal. In Lemma 4 the projective limit representation of $\mathscr{S}\left\{M_{p}\right\}$ is given. In these lemmas conditions (M.1) and (M.3) $^{\prime}$ are assumed. In other assertions conditions (M.1), (M.2), and (M.3) are assumed. Theorems 1 and 2 are the so-called second structural theorems for bounded sets in spaces $\mathscr{D}_{L^{\prime}}^{\left\{M_{p}\right\}}, t \in[1, \infty]$, and $\mathscr{S}^{\prime *}$ and for equicontinuous sets $\widetilde{\mathscr{D}}_{L^{t}}^{\prime\left\{M_{p}\right\}}, t \in[1, \infty]$. These theorems are based on the parameterixes for appropriate ultradifferential operators constructed in [6] (Lemma 5). In Theorem 3(i) it is proved that a set $B \subset \mathscr{D}^{\prime\left(M_{p}\right)}$ (resp. $B \subset \mathscr{D}^{\prime\left(M_{p}\right)}$ ) is bounded if for every $\phi \in \mathscr{D}^{\prime\left(M_{p}\right)}$ (resp. $\phi \in \mathscr{D}^{\left(M_{p}\right)}$ ) and every bounded open set $\Omega$, $\sup \{|(f * \phi)(x)| ; f \in B, x \in \Omega\}<\infty$. In Theorem 3(ii) an improvement of the quoted assertion is given, and in Theorem 4 the corresponding characterizations of a convergent sequence in $\mathscr{D}^{\prime\left(M_{p}\right)}$ (resp. $\left.\mathscr{D}^{\prime}\left\{M_{p}\right\}\right)$ are given.

\section{NotATION AND NOTIONS}

The sets of real, complex, and natural numbers are denoted by $\mathbf{R}, \mathbf{C}, \mathbf{N}$;

Received by the editors July 27, 1992.

1991 Mathematics Subject Classification. Primary 46F05. 
$\mathbf{N}_{0}=\mathbf{N} \cup\{0\}$.

$$
\begin{gathered}
\langle x, y\rangle=\sum_{i=1}^{n} x_{i} y_{i}, \quad x^{\alpha}=x_{1}^{\alpha_{1}} \cdots x_{n}^{\alpha_{n}}, \quad|\alpha|=\alpha_{1}+\cdots+\alpha_{n}, \quad h^{\alpha}=h^{|\alpha|}, \\
\alpha !=\alpha_{1}, \ldots, \alpha !, \quad D_{t}=\frac{1}{i} \frac{d}{d t}, \quad D^{\alpha}=D_{x_{1}}^{\alpha_{1}} \cdots D_{x_{n}}^{\alpha_{n}}, \quad \partial^{\alpha}=\frac{\partial^{\alpha_{1}+\cdots+\alpha_{n}}}{\partial x_{1}^{\alpha_{1}} \cdots \partial x_{n}^{\alpha_{n}}},
\end{gathered}
$$

where $x, y \in \mathbf{R}^{n}, \alpha \in \mathbf{N}_{0}^{n}, h>0, t \in \mathbf{R}$.

By $L^{s}, s \in[1, \infty]$, we denote the well-known space of functions $f$ (classes) for which $|f|^{s}$ is integrable on $\mathbf{R}^{n}$. The norm in this space is denoted by \|\|$_{L^{s}}$; $C^{\infty}$ is the space of smooth functions on $\mathbf{R}^{n}$. For an $f \in L^{1}$ the Fourier transform is defined by

$$
\mathscr{F} f(\xi)=\widehat{f}(\xi)=\int_{\mathbf{R}^{n}} e^{-\langle x, \xi\rangle} f(x) d x, \quad \xi \in \mathbf{R}^{n} .
$$

By $M_{p}, p \in \mathbf{N}_{0}$, we denote a sequence of positive numbers. Assume $M_{0}=$ 1. The following conditions on these sequences will be considered.

(M.1) $M_{p}^{2} \leq M_{p-1} M_{p+1}, p \in \mathbf{N}$;

(M.2) $\quad M_{p} \leq A H^{p} M_{p-1}, p \in \mathbf{N}$, for some $A>0, H>0$;

(M.2) $M_{p} \leq A H^{p} M_{q} M_{p-q}, 0 \leq q \leq p, p \in \mathbf{N}$, for some $A>0, H>0$;

(M.3) $\sum_{q=1}^{\infty} M_{q-1} / M_{q}<\infty$;

(M.3) $\sum_{q=p+1}^{\infty} M_{q-1} / M_{q} \leq A p M_{p} / M_{p+1}, p \in \mathbf{N}$, for some $A>0$.

For the properties of such sequences we refer to [4] and [9]. In the definitions of test function spaces which are to follow we shall always assume that (M.1) and (M.3)' hold.

Recall the definition of Beurling and Roumieau spaces of ultradifferentiable functions [4]. If $K$ is a compact subset of $\mathbf{R}^{n}, h>0$, and $\phi \in C^{\infty}$, then

$$
\|\phi\|_{K, h}=\sup \left\{\frac{\left|\partial^{\alpha} \phi(x)\right|}{h^{\alpha} M_{|\alpha|}} ; \alpha \in \mathbf{N}_{0}^{n}, x \in K\right\} .
$$

Denote by $\mathscr{D}_{K, h}^{M_{p}}$ the space of functions $\phi$ from $C^{\infty}$ for which $\operatorname{supp} \phi \subset K$ and $\|\phi\|_{K, h}<\infty$. The basic spaces of functions of classes $\left(M_{p}\right)$ and $\left\{M_{p}\right\}$ are defined by

$$
\begin{array}{ll}
\mathscr{D}_{K}^{\left(M_{p}\right)}=\text { proj } \lim _{h \rightarrow 0} \mathscr{D}_{K, h}^{M_{p}}, & \mathscr{D}_{K}^{\left\{M_{p}\right\}}=\text { ind } \lim _{h \rightarrow \infty} \mathscr{D}_{K, h}^{M_{p}}, \\
\mathscr{D}^{\left(M_{p}\right)}=\text { ind } \lim _{K \subset \subset \mathbf{R}^{n}} \mathscr{D}_{K}^{\left(M_{p}\right)}, & \mathscr{D}^{\left\{M_{p}\right\}}=\text { ind } \lim _{K \subset \subset \mathbf{R}^{n}} \mathscr{D}_{K}^{\left\{M_{p}\right\}} .
\end{array}
$$

The notation $K \subset \subset \mathbf{R}^{n}$ means that $K$ is compact and "grows" up to $\mathbf{R}^{n}$. For the properties of basic spaces which correspond to a sequence $M_{p}$ which satisfies given conditions we refer to [4].

Denote by $\mathfrak{R}$ the set of all positive sequences $r_{p}, p \in \mathbf{N}$, which increase to $\infty$. This set is partially ordered and directed by the relation $r_{p} \preceq s_{p}$ defined by $r_{p} \leq s_{p}, p>p_{0}$, for some $p_{0}$.

Let $r_{p} \in \mathfrak{R}$ and $K$ be a compact set in $\mathbf{R}^{n}$. Denote by $\mathscr{D}_{K, r_{p}}^{\left\{M_{p}\right\}}$ the space of smooth functions $\varphi$ on $\mathbf{R}^{n}$ supported by $K$ such that

$$
\|\varphi\|_{K, r_{p}}=\sup \left\{\frac{\left|\partial^{\alpha} \varphi(x)\right|}{M_{|\alpha|}\left(\prod_{i=1}^{|\alpha|} r_{i}\right)}, \alpha \in \mathbf{N}_{0}^{n}, \quad x \in K\right\}<\infty .
$$


Clearly, this a Banach space. It is proved in [4] that under conditions (M.1), (M.2), and (M.3)

$$
\mathscr{D}_{K}^{\left\{M_{p}\right\}}=\operatorname{proj} \lim _{r_{p} \in \Omega} \mathscr{D}_{K, r_{p}}^{\left\{M_{p}\right\}}
$$

Let $\Omega$ be a bounded open set in $\mathbf{R}^{n}$. We put

$$
\mathscr{D}_{\Omega, r}^{\left(M_{p}\right)}=\text { ind } \lim _{K \subset \subset \Omega} \mathscr{D}_{K, r}^{M_{p}}, \quad \mathscr{D}_{\Omega, r_{p}}^{\left\{M_{p}\right\}}=\text { ind } \lim _{K \subset \subset \Omega} \mathscr{D}_{K, r_{p}}^{\left\{M_{p}\right\}} .
$$

These spaces will be used in Theorems 1 and 2 .

Let $s \in[1, \infty]$. We define

$$
\mathscr{D}_{L^{s}}^{\left(M_{p}\right)}=\operatorname{proj} \lim _{h \rightarrow 0} \mathscr{D}_{L^{s}, h}^{M_{p}}, \quad \mathscr{D}_{L^{s}}^{\left\{M_{p}\right\}}=\text { ind } \lim _{h \rightarrow \infty} \mathscr{D}_{L^{s}, h}^{M_{p}},
$$

where $\mathscr{D}_{L^{s}, h}^{M_{p}}$ is the space of functions $\phi$ from $C^{\infty}$ equipped with the norm

$$
\|\phi\|_{L^{s}, h}=\sup \left\{\frac{\left\|\partial^{\alpha} \phi\right\|_{L^{s}}}{h^{\alpha} M_{|\alpha|}} ; \alpha \in \mathbf{N}_{0}^{n}\right\}<\infty .
$$

The space $\mathscr{D}_{L^{s}}^{\left(M_{p}\right)}$ is defined in [10].

The common notation for the symbols $\left(M_{p}\right)$ and $\left\{M_{p}\right\}$ will be $*$. If $\Omega$ is an open set in $\mathbf{R}^{n}$ and $K$ is a compact subset of $\Omega$, then $\mathscr{D}_{K}^{*}(\Omega)$ and $\mathscr{D}^{*}(\Omega)$ are defined analogously to the case $\Omega=\mathbf{R}$.

Since $\mathscr{D}^{*}$ is dense in $\mathscr{D}_{L^{s}}^{*}, s \in[1, \infty)$, and the inclusion mapping is continuous, it follows that the corresponding strong duals of $\mathscr{D}_{L^{s}}^{*}$ and $\mathscr{D}_{L^{i}}^{\prime *}$, $t=s /(s-1)$, are subspaces of Beurling and Roumieau ultradistribution spaces. We denote by $\dot{\mathscr{B}}^{*}$ the completion of $\mathscr{D}^{*}$ in $\mathscr{D}_{L^{\infty}}^{*}$. The strong dual of $\dot{\mathscr{B}}^{*}$ is denoted by $\mathscr{D}_{L^{1}}^{\prime *}$.

The space $\mathscr{S}^{*}$ is defined as follows [7, 8]. Denote by $S_{h}^{M_{p}}, h>0$, the space of functions $\phi$ from $C^{\infty}$ such that

$$
\gamma_{h}(\phi)=\sup \left\{\frac{\left(1+|x|^{2}\right)^{\alpha / 2}\left\|\partial^{\beta} \phi(x)\right\|_{L^{2}}}{h^{\alpha+\beta} M_{|\alpha|} M_{|\beta|}} ; \alpha, \beta \in \mathbf{N}_{0}^{n}\right\}<\infty .
$$

The Hermite expansion of elements from $\mathscr{S}^{*}$ is determined in [8] under assumptions (M.1), (M.2), and (M.3)', which imply that for $k>h$ the inclusion mapping $\mathscr{S}_{h}^{M_{p}} \rightarrow \mathscr{S}_{k}^{M_{p}}$ is nuclear. Thus,

$$
S^{\left(M_{p}\right)}=\operatorname{proj} \lim _{h \rightarrow 0} S_{h}^{M_{p}} \quad\left(S^{\left\{M_{p}\right\}}=\text { ind } \lim _{h \rightarrow \infty} S_{h}^{M_{p}}\right)
$$

is (FN) space ((LN) space) under conditions (M.1), (M.2), and (M.3)' .

Note that conditions (M.1) and (M.3)' imply that the inclusion mapping $S_{h}^{M_{p}} \rightarrow S_{k}^{M_{p}}, k>h$, is compact [7].

The space $S^{\left\{!^{\alpha}\right\}}$ is the Gelfand-Shilov-type space [2], and for $S^{\left(p^{\alpha}\right)}, \alpha>1$, we refer to [10]. $\mathscr{D}^{*}$ is dense in $S^{*}$, and, with the assumptions (M.1), (M.2), and (M.3) $)^{\prime}, S^{*}$ is invariant under the Fourier transformation. So the same holds for the strong dual $S^{\prime *}$.

An operator of the form $P(D)=\sum_{\alpha \in \mathbf{N}_{0}^{n}} a_{\alpha} D^{\alpha}, a_{\alpha} \in \mathbf{C}$, is an (ultradifferential) operator of class $\left(M_{p}\right)$ (resp. of class $\left\{M_{p}\right\}$ ) if there are constants $A>0$, $h>0$ (resp. for every $h>0$ there is $A>0$ ), such that

$$
\left|a_{\alpha}\right| \leq A h^{\alpha} / M_{|\alpha|}, \quad \alpha \in \mathbf{N}_{0}^{n} .
$$


Special classes of entire functions [4] will be used. We recall some facts from [5]. In the sequel $n^{\prime}$ will be an integer greater than $n / 2$ and $m_{p}=M_{p} / M_{p-1}$, $p \in \mathbf{N}$. Let $r>0$. Put

(1)

$P_{r}(\zeta)=\left(1+\zeta_{1}^{2}+\cdots+\zeta_{n}^{2}\right)^{n^{\prime}} \prod_{i=1}^{\infty}\left(1+\frac{\zeta_{1}^{2}+\cdots+\zeta_{n}^{2}}{r^{2} m_{i}^{2}}\right), \quad \zeta=\left(\zeta_{1}, \ldots, \zeta_{n}\right) \in \mathbf{C}^{n}$

If (M.1), (M.2), and (M.3) hold, then $P_{r}(D)$ is an ultradifferentiable operator of class $\left(M_{p}\right)$; it maps $\mathscr{D}^{\left(M_{p}\right)}$ into itself, and

$$
\mathscr{F}\left(P_{r}(D) \phi\right)(\xi)=P_{r}(\xi) \hat{\phi}(\xi), \quad \xi \in \mathbf{R}^{n}, \phi \in \mathscr{D}^{\left(M_{p}\right)} .
$$

Put

$$
P_{r_{p}}(\zeta)=\left(1+\zeta_{1}^{2}+\cdots+\zeta_{1}^{2}\right)^{n^{\prime}} \prod_{i=1}^{\infty}\left(1+\frac{\zeta_{1}^{2}+\cdots+\zeta_{n}^{2}}{r_{i}^{2} m_{i}^{2}}\right), \quad \zeta \in \mathbf{C}^{n}
$$

Under conditions (M.1), (M.2), and (M.3) it is of class $\left\{M_{p}\right\}$. For elements of $\mathscr{D}\left\{M_{p}\right\}$ and $P_{r_{p}}(D)$ equality (2) holds, as well.

The associated function for

$$
N_{p}=M_{p}\left(\prod_{i=1}^{p} r_{i}\right), \quad p \in \mathbf{N}
$$

is defined by

$$
N(\rho)=\sup \left\{\ln \frac{\rho^{p} 1}{N_{p}} ; p \in \mathbf{N}_{0}^{n}\right\}, \quad \rho>0 .
$$

If an element of $\mathfrak{R}$ is denoted by $\tilde{r}_{p}$, the corresponding associated function is denoted by $\widetilde{N}$. For the sequence $M_{p}$ the associated function is denoted by $M$. From the definition it follows that for every $r_{p} \in \mathfrak{R}, C>0$, and $c>0$ there are $\tilde{r}_{p} \in \mathfrak{R}$ and $\rho_{0}>0$ such that

$$
C N(c \rho) \leq \tilde{N}(\rho), \quad \rho>\rho_{0} .
$$

Assume (M.1), (M.2), and (M.3). From [4, Proposition 4.5 and p. 91] it follows that there exist $D>0$ and $c>0$ such that

$$
D \exp (-N(c|\xi|)) \leq\left|1 / P_{r_{p}}(\xi)\right| \leq \exp (-N(\xi)), \quad \xi \in \mathbf{R}^{n} .
$$

By using the Cauchy formula

$$
\partial^{k}\left(1 / P_{r_{p}}(\xi)\right)=\frac{k !}{(2 \pi i)^{n}} \int_{\Gamma_{1}} \cdots \int_{\Gamma_{n}} \frac{\left(1 / P_{r_{p}}\right)(\zeta) d \zeta_{1} \cdots d \zeta_{n}}{\left(\zeta_{1}-\xi_{1}\right)^{k_{1}+1} \cdots\left(\zeta_{n}-\xi_{n}\right)^{k_{n}+1}}, \quad k \in \mathbf{N}_{0}^{n},
$$

where $\Gamma_{i}=\left\{\zeta_{i}\left|\zeta_{i}-\xi_{i}\right|=d\right\}, i=1, \ldots, n, d>0, \xi \in \mathbf{R}^{n}$, we obtain that there exists $C>0$ such that

$$
\left|\partial^{k}\left(1 / P_{r_{p}}(\xi)\right)\right| \leq C k ! d^{-k} \exp (-N(\xi) / C), \quad \xi \in \mathbf{R}^{n} .
$$

The following two-dimensional version of [5, Lemma 3.4] is needed. 
Lemma 1. Let $a_{p, q}>0, p, q \in \mathbf{N}_{0}$.

(i) There are $h>0$ and $C>0$ such that

$$
\sup \left\{a_{p, q} / h^{p+q} ; p, q \in \mathbf{N}_{0}\right\} \leq C
$$

if and only if for every sequence $r_{i}, s_{j}$ from $\mathfrak{R}$

$$
\sup \left\{\frac{a_{p, q}}{\left(\prod_{i=1}^{p} r_{i} \prod_{j=1}^{q} s_{j}\right)} ; p, q \in \mathbf{N}_{0}\right\}<\infty .
$$

(ii) There are sequences $r_{i}, s_{j} \in \mathfrak{R}$ and $C>0$ such that

$$
\sup \left\{\left(\prod_{i=1}^{p} r_{i} \prod_{j=1}^{q} s_{j}\right) a_{p, q} ; p, q \in \mathbf{N}_{0}\right\} \leq C
$$

if and only if for every $h>0$

$$
\sup \left\{h^{p+q} a_{p, q} ; p, q \in \mathbf{N}_{0}\right\}<\infty .
$$

Proof. One only has to prove the if parts.

(i) Assume that for every $r_{i}, s_{j} \in \mathfrak{R}$ (8) holds but for every $h>0$ and $C>0$ (7) does not hold. Let $h_{\nu}$ be a sequence which strictly increases to $\infty$. There exists a sequence $\left(p_{\nu}, q_{\nu}\right)$ from $\mathbf{N}_{0}^{2}$ such that

$$
p_{\nu+1}+q_{\nu+1}>p_{\nu}+q_{\nu} \quad \text { and } \quad h^{-\left(p_{\nu}+q_{\nu}\right)} a_{p_{\nu}, q_{\nu}}>\nu, \quad \nu \in \mathbf{N} .
$$

The following cases may appear:

(a) There is $p_{0} \in \mathbf{N}_{0}$ such that $\left(p_{0}, q_{k}\right)$ is a subsequence of $\left(p_{\nu}, q_{\nu}\right)$ and $q_{k}$ is strictly increasing.

(b) Symmetric case to previous one.

(c) There is a subsequence $\left(p_{k}, q_{k}\right)$ of $\left(p_{\nu}, q_{\nu}\right)$ such that both $p_{k}$ and $q_{k}$ are strictly increasing.

Let us prove that $(\mathrm{c})$ implies the contradiction. The other two cases are similar. Let

$$
\begin{gathered}
r_{i}=h_{1}, \quad 1 \leq i \leq p_{1}, \quad s_{j}=h_{1}, \quad 1 \leq j \leq q_{1} \\
r_{i}=\left(h_{k}^{p_{k}} h_{k-1}^{-p_{k-1}}\right)^{1 /\left(p_{k}-p_{k-1}\right)}, \quad p_{k-1}<i \leq p_{k} \\
s_{j}=\left(h_{k}^{q_{k}} h_{k-1}^{-q_{k-1}}\right)^{1 /\left(q_{k}-q_{k-1}\right)}, \quad q_{k-1}<j<q_{k}, k=2,3, \ldots .
\end{gathered}
$$

The constructed sequences $r_{i}$ and $s_{j}$ do not satisfy (8), and this is a contradiction.

(ii) Put

$$
\begin{aligned}
& b_{\nu}=\sup \left\{a_{p, q} ; p+q=\nu, p, q \in \mathbf{N}_{0}\right\}, \quad \nu \in \mathbf{N}_{0} ; \\
& C_{h}=\sup \left\{h^{\nu} b_{\nu} ; \nu \in \mathbf{N}_{0}\right\}, \quad h \geq 1 ; \\
& \widetilde{C}_{h}=\sup \left\{h^{p+q} a_{p, q} ; p, q \in \mathbf{N}_{0}\right\}, \quad h \geq 1, \\
& H_{\nu}=\sup \left\{h^{\nu} C_{h}^{-1} ; h \geq 1\right\}, \quad \nu \in \mathbf{N}_{0} .
\end{aligned}
$$

Clearly $\widetilde{C}_{h} \leq C_{h}$. Fix $p, q \in \mathbf{N}_{0}$, and let $\nu=p+q$. For every $h \geq 1$,

$$
\sup \left\{h^{\nu} a_{p, q} / C_{h} ; h \geq 1\right\} \leq \sup \left\{\widetilde{C}_{h} / C_{h} ; h \geq 1\right\} \leq 1 \text {. }
$$


Thus, $\sup \left\{H_{p+q} a_{p, q} ; p, q \in \mathbf{N}_{0}\right\} \leq 1$. Put $h_{i}=H_{i} / H_{i-1}, i \in \mathbf{N}$. The sequence $h_{i}$ is increasing, and, for every $h>0, H_{p} / h^{p} \rightarrow \infty, p \rightarrow \infty$. Since

$$
\left(\prod_{i=1}^{p} h_{i} \prod_{j=1}^{q} h_{j}\right) a_{p, q} \leq \prod_{i=1}^{p+q} h_{i} a_{p, q},
$$

by taking $r_{i}=h_{i}, s_{j}=h_{j}$, we obtain

$$
\left(\prod_{i=1}^{p} r_{i} \prod_{j=1}^{q} s_{j}\right) a_{p, q}<\infty,
$$

and this implies the assertion.

\section{StRUCTURAL THEOREMS}

Denote by $C_{0}$ the space of continuous functions $f$ on $\mathbf{R}^{n}$ such that $\lim _{|x| \rightarrow \infty} f(x)=0$ equipped with the norm \|\|$_{L^{\infty}}$. Its dual space, the space of measures, is denoted by $\mathscr{M}^{1}$ (as in [3]), and we denote the dual norm in $\mathscr{M}^{1}$ by \|\|$_{\mathscr{K}^{1}}$. Note that, under conditions (M.1) and (M.3) ${ }^{\prime}, \mathscr{D}^{*}$ is dense in $C_{0}$.

Lemma 2. Let $M_{p}$ satisfy (M.1) and (M.3)'.

(i) $A$ set $B \subset \mathscr{D}_{L^{i}}^{\prime\left\{M_{p}\right\}}, t \in(1, \infty]$, is bounded if and only if every $f \in B$ can be represented in the form

$$
f=\sum_{|\alpha|=0}^{\infty} D^{\alpha} f_{\alpha}, \quad \text { where } f_{\alpha} \in L^{t}, \alpha \in \mathbf{N}^{n},
$$

such that for some $d>0$ there exists $C>0$ independent of $f \in B$ such that

$$
\sum_{|\alpha|=0}^{\infty} d^{\alpha} M_{|\alpha|}\left\|f_{\alpha}\right\|_{L^{t}}<C
$$

(ii) $A$ set $B \subset \mathscr{D}_{L^{1}}^{\prime\left\{M_{p}\right\}}$ is bounded if and only if the representation of $f$ in (i) holds with $f_{\alpha} \in \mathscr{M}^{1}$ and the condition in (i) holds with the norm $\left\|f_{\alpha}\right\|_{\mathscr{M}_{1}}$.

(iii) Let $f \in \mathscr{D}^{\prime}\left\{M_{p}\right\}$. It belongs to $\mathscr{D}_{L^{t}}^{\prime\left\{M_{p}\right\}}, t \in[1, \infty]$, if and only if $f$ is of the form $f=\sum_{|\alpha|=0}^{\infty} D^{\alpha} f_{\alpha}$, where $f_{\alpha} \in L^{t}$ if $t \in(1, \infty]$ and $f_{\alpha} \in \mathscr{M}^{1}$ if $t=1, \alpha \in \mathbf{N}_{0}^{n}$, such that for every $d>0$

$$
\begin{cases}\sum_{|\alpha|=0}^{\infty} d^{\alpha} M_{|\alpha|}\left\|f_{\alpha}\right\|_{L^{t}}<\infty & \text { if } t \in(1, \infty] \\ \sum_{|\alpha|=0} d^{\alpha} M_{|\alpha|}\left\|f_{\alpha}\right\|_{\mathscr{M}^{1}}<\infty & \text { if } t=1\end{cases}
$$

Proof. Clearly, the conditions given in (i)-(iii) are sufficient, so, we will prove that they are necessary.

(i) Since $\mathscr{D}_{L^{s}}^{\left(M_{p}\right)}, s=t /(t-1) \in[1, \infty)$, is barrelled, $B$ is an equicontinuous set in $\mathscr{D}_{L^{t}}^{\prime\left(M_{p}\right)}$, and for some $d>0$ and $C>0$

$$
|\langle f, \phi\rangle| \leq C\|\phi\|_{L^{s}, d}, \quad \phi \in \mathscr{D}_{L^{p}}^{\left(M_{p}\right)}, f \in B .
$$

Hence, by Hahn-Banach Theorem, $B$ can be extended to an equicontinuous set $B_{1}$ on $\mathscr{D}_{L^{s}, d}^{M_{p}}$. Let $Y_{s, d}$ be the space of sequences $\left(\phi_{\alpha}\right)$ from $L^{s}$ such that

$$
\left\|\left(\phi_{\alpha}\right)\right\|_{L^{s}, d}=\sup \left\{\frac{\left\|\phi_{\alpha}\right\|_{L^{s}}}{d^{\alpha} M_{|\alpha|}} ; \alpha \in \mathbf{N}_{0}^{n}\right\}<\infty
$$


equipped with this norm. Again, by Hahn-Banach Theorem, $B_{1}$ can be extended to an equicontinuous set $B_{2}$ on $Y_{s, d}$. An equicontinuous set on $Y_{s, d}$ consists of sequences $\left(f_{\alpha}\right)$ from $L^{t}$ for which (12) holds, and this implies assertion (i).

(ii) Let $X_{\infty, h}$ be the space of smooth functions $\phi$ such that, for every $\alpha \in$ $\mathbf{N}_{0}^{n}, \phi^{(\alpha)} \in C_{0}$ and $\|\phi\|_{L^{\alpha}, h}<\infty$ equipped with the norm \|\|$_{L^{\infty}, h}$. There holds

$$
\dot{\mathscr{B}}^{\left(M_{p}\right)}=\text { proj } \lim _{h \rightarrow \infty} X_{\infty, h},
$$

which implies that $\dot{\mathscr{B}}^{\left(M_{p}\right)}$ is barrelled. Thus in the same way as in (i) the proof of (ii) follows.

(iii) Let $Y_{s, h}, s \in[1, \infty], h>0$, be the space of sequences $\left(\phi_{\alpha}\right), \alpha \in \mathbf{N}_{0}^{n}$, from $L^{s}$ and $s \in[1, \infty)$ and from $C_{0}$ for $s=\infty$ such that

$$
\left\|\left(\phi_{\alpha}\right)\right\|_{L^{s}, h}=\sup \left\{\frac{\left\|\phi_{\alpha}\right\|_{L^{s}}}{h^{\alpha} M_{|\alpha|}} ; \alpha \in \mathbf{N}_{0}^{n}\right\}<\infty
$$

equipped with the given norm.

Let $X_{s, h}=\mathscr{D}_{s, h}^{M_{p}}, s \in[1, \infty), h>0$, and $X_{\infty, h}$ be as in the proof of (ii). We identify $X_{s, h}$ with the corresponding subspace of $Y_{s, h}, s \in[1, \infty], h>0$, via the mapping $\phi \rightarrow\left(\phi^{(\alpha)}\right)$. Note that $\dot{\mathscr{B}}^{\left\{M_{p}\right\}}=$ ind $\lim _{h \rightarrow \infty} X_{s, h}$. With the given identification we have

$$
\begin{aligned}
& \mathscr{D}_{L^{s}}^{\left\{M_{p}\right\}} \subset Y_{s}=\text { ind } \lim _{h \rightarrow \infty} Y_{s, h}, \quad s \in[1, \infty), \\
& \dot{\mathscr{B}}^{\left\{M_{p}\right\}} \subset Y_{\infty}=\text { ind } \lim _{h \rightarrow \infty} Y_{\infty, h} .
\end{aligned}
$$

Since the inclusion mappings are continuous, a continuous linear functional on $\mathscr{D}_{L^{s}}^{\left\{M_{p}\right\}}$ or $\dot{\mathscr{B}}\left\{M_{p}\right\}$ is continuous on this space equipped with the induced topology from ind $\lim _{h \rightarrow \infty} Y_{s, h}, s \in[1, \infty]$. Thus, Hahn-Banach Theorem implies assertion (iii) because in the set-theoretical sense we have

$$
\left(\text { ind } \lim _{h \rightarrow \infty} Y_{s, h}\right)^{\prime}=\operatorname{proj} \lim _{h \rightarrow \infty} Y_{s, h}^{\prime}, \quad s \in[1, \infty] .
$$

Remark. With the notation as in (iii), for $s \in(1, \infty)$, the sequence $Y_{s, h}, h \in$ $\mathbf{N}$, is weakly compact. This implies that $X_{s, h}, h \in \mathbf{N}$, and $Z_{s, h}=Y_{s, h} / X_{x, h}$, $h \in \mathbf{N}$, are weakly compact as well, and thus the dual Mittag-Leffler Lemma [4, Lemma 1.4] implies that the sequence

$$
0 \leftarrow \operatorname{proj} \lim _{h \rightarrow \infty} X_{s, h}^{\prime} \leftarrow \operatorname{proj} \lim _{h \rightarrow \infty} Y_{s, h}^{\prime}
$$

is exact, where (in the topological sense)

$$
\begin{aligned}
& \text { proj } \lim _{h \rightarrow \infty} X_{s, h}^{\prime}=X_{s}^{\prime}=\left(\text { ind } \lim _{h \rightarrow \infty} X_{s, h}\right)^{\prime}, \\
& \text { proj } \lim _{h \rightarrow \infty} Y_{s, h}^{\prime}=Y_{s}^{\prime}=\left(\text { ind } \lim _{h \rightarrow \infty} Y_{s, h}\right)^{\prime} .
\end{aligned}
$$

This implies that $\mathscr{D}_{L^{s}}^{\left\{M_{p}\right\}}$ and $X_{s}$ equipped with the induced topology from $Y_{s}$ have the same strong duals [4, Lemma 1.4(iii)]. We do not know whether the 
space $X_{s}$ with the induced topology is quasi-barrelled, and because of that we do not have the characterization of a bounded set in $\mathscr{D}_{L^{t}}^{\prime\left\{M_{p}\right\}}, t \in(1, \infty)$.

Denote by $\mathscr{D}_{L^{s}, r_{p}}^{\left\{M_{p}\right\}}, r_{p} \in \mathfrak{R}, s \in[1, \infty]$, the space of smooth functions $\varphi$ such that

$$
\|\varphi\|_{L^{s}, r_{p}}=\sup \left\{\frac{\left\|\partial^{\alpha} \varphi\right\|_{L^{s}}}{M_{|\alpha|}\left(\prod_{i=1}^{|\alpha|} r_{i}\right)} ; \alpha \in \mathbf{N}_{0}^{n}\right\}<\infty
$$

equipped with this norm, and let $\widetilde{\mathscr{D}}_{L^{s}}^{\left\{M_{p}\right\}}=\operatorname{proj} \lim _{r_{p} \in \mathfrak{R}} \mathscr{D}_{L^{s}, r_{p}}^{\left\{M_{p}\right\}}$. The completion of $\mathscr{D}\left\{M_{p}\right\}$ with respect to $\widetilde{\mathscr{D}}_{L^{\infty}}^{\left\{M_{p}\right\}}$ is denoted by $\dot{\mathscr{\mathscr { B }}}^{\left\{M_{p}\right\}}$. The corresponding dual spaces are denoted by $\widetilde{\mathscr{D}}_{L^{t}}^{\prime\left\{M_{p}\right\}}, t=s /(s-1) \in(1, \infty]$, and $\widetilde{\mathscr{D}}_{L^{1}}^{\prime\left\{M_{p}\right\}}$.

Lemma 3. Let $M_{p}$ satisfy (M.1) and (M.3)'.

(i) $\widetilde{\mathscr{D}}_{L^{s}}^{\left\{M_{p}\right\}}=\mathscr{D}_{L^{s}}^{\left\{M_{p}\right\}}, s \in(1, \infty)$, in the set-theoretical sense. The same holds for $\dot{\mathscr{B}}\left\{M_{p}\right\}$ and $\dot{\mathscr{\mathscr { B }}}\left\{M_{p}\right\}$

(ii) The inclusion mappings $i: \mathscr{D}_{L^{s}}^{\left\{M_{p}\right\}} \rightarrow \widetilde{\mathscr{D}}_{L^{s}}^{\left\{M_{p}\right\}}, s \in[1, \infty)$, and $i: \dot{\mathscr{B}}\left\{M_{p}\right\}$ $\rightarrow \dot{\widetilde{\mathscr{B}}}^{\left\{M_{p}\right\}}$ are continuous.

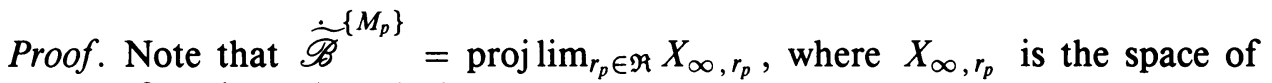
smooth functions $\phi$ such that

$$
\phi^{(\alpha)} \in C_{0}, \quad \alpha \in \mathbf{N}_{0}^{n}, \quad\|\phi\|_{L^{\infty}, r_{p}}<\infty
$$

equipped with this norm.

The proof of (i) follows from [5, Lemma 3.4], and (ii) follows from the inequality

$$
\|\phi\|_{L^{s}, r_{p}} \leq C_{r_{p}, h}\|\phi\|_{L^{s}, h}, \quad \phi \in \mathscr{D}_{L^{s}, h}^{\left\{M_{p}\right\}},
$$

where $r_{p} \in \mathfrak{R}, h>0$, and $C_{r_{p}, h}$ is a suitable constant.

Lemma 4. Let $M_{p}$ satisfy (M.1) and (M.3)'.

(i) $A$ set $B \subset \mathscr{S}^{\prime\left(M_{p}\right)}$, resp. $\mathscr{S}^{\prime}\left\{M_{p}\right\}$, is bounded if and only if every $f \in B$ can be represented in the form

$$
f=\sum_{\substack{|\alpha|=0 \\|\beta|=0}}^{\infty} D^{\alpha}\left(\left(1+|x|^{2}\right)^{\beta / 2} f_{\alpha, \beta}\right), \quad \text { where } f_{\alpha, \beta} \in L^{2}, \alpha, \beta \in \mathbf{N}_{0}^{n},
$$

such that for some $d>0$ (resp. every $d>0$ ) there exists $D>0$ independent of $f \in B$ such that

$$
\sum_{\substack{|\alpha|=0 \\|\beta|=0}}^{\infty} d^{\alpha+\beta} M_{|\alpha|} M_{|\beta|}\left\|f_{\alpha, \beta}\right\|_{L^{2}}<D
$$

(ii) $\mathscr{S}^{\left\{M_{p}\right\}}=\operatorname{proj} \lim _{r_{i}, s_{j} \in \mathfrak{R}} \mathscr{S}_{r_{i}, s_{j}}^{M_{p}}$ where $\mathscr{S}_{r_{i}, s_{j}}^{M_{p}}$ is the space of functions $\varphi$ from $C^{\infty}$ such that

$$
\gamma_{r_{i}, s_{j}}(\varphi)=\sup \left\{\frac{\left\|\left(1+|x|^{2}\right)^{\beta / 2} \partial^{\alpha} \varphi\right\|_{L^{2}}}{M_{|\alpha|}\left(\prod_{i=1}^{|\alpha|} r_{i}\right) m_{|q|}\left(\prod_{j=1}^{|\beta|} s_{j}\right)} ; \alpha, \beta \in \mathbf{N}_{0}^{n}\right\}<\infty .
$$


Proof. (i) We shall prove the assertion in the $\left\{M_{p}\right\}$ case because it is rather complicated. Note that $\mathscr{S}^{\left\{M_{p}\right\}}$ is barrelled, and thus $B$ is an equicontinuous subset of $\mathscr{S}^{\prime}\left\{M_{p}\right\}$.

Let $W_{h}, h>0$, be the space of sequences $\left(\phi_{\alpha, \beta}\right), \alpha, \beta \in \mathbf{N}_{0}^{n}$, from $L^{2}$ such that

$$
\left\|\left(\phi_{\alpha, \beta}\right)\right\|_{L^{s}, h}=\sup \left\{\frac{\left\|\phi_{\alpha, \beta}\right\|_{L^{2}}}{h^{\alpha+\beta} M_{|\alpha|} M_{|\beta|}} ; \alpha, \beta \in \mathbf{N}_{0}^{n}\right\}<\infty
$$

equipped with this norm. We identify $\mathscr{S}_{h}^{M_{p}}$ by the corresponding subspace of $W_{h}$. Since $W_{h}, h \in \mathbf{N}$, is a weakly compact inductive sequence and $\mathscr{S}_{h}^{M_{p}}$, $h \in \mathbf{N}$, is a compact one, [4, Lemma 1.4(iii)] implies that the sequence

$$
0 \leftarrow \text { proj } \lim _{h \rightarrow \infty}\left(\mathscr{S}_{h}^{M_{p}}\right)^{\prime} \leftarrow \operatorname{proj} \lim _{h \rightarrow \infty} W_{h}^{\prime}
$$

is exact, where

$$
\begin{gathered}
\operatorname{proj} \lim _{h \rightarrow \infty}\left(\mathscr{S}_{h}^{M_{p}}\right)^{\prime}=\mathscr{S}^{\prime\left\{M_{p}\right\}}=\left(\text { ind } \lim _{h \rightarrow \infty} \mathscr{S}_{h}^{M_{p}}\right)^{\prime}, \\
\operatorname{proj} \lim _{h \rightarrow \infty} W_{h}^{\prime}=W^{\prime}=\left(\text { ind } \lim _{h \rightarrow \infty} W_{h}\right)^{\prime} .
\end{gathered}
$$

Since $\mathscr{S}^{\left\{M_{p}\right\}}$ is a Montel space by [4, Lemma 1.4(v)], $\mathscr{S}\left\{M_{p}\right\}$ is a closed subspace of $W$, and by Hahn-Banach Theorem the equicontinuous set $B \subset$ $\mathscr{S}^{\prime}\left\{M_{p}\right\}$ can be extended to the equicontinuous set $\widetilde{\mathscr{B}}$ in $W^{\prime}$. Thus $\widetilde{B}$ consists of the sequence $\left(f_{\alpha, \beta}\right), \alpha, \beta \in \mathbf{N}_{0}^{n}$, from $L^{2}$ such that for every $d \in \mathbf{N}$ there is $C>0$ which is the same for all the elements from $\widetilde{B}$ such that

$$
\sum_{\substack{|\alpha|=0 \\|\beta|=0}}^{\infty} d^{\alpha+\beta} M_{|\alpha|} M_{|\beta|}\left\|f_{\alpha, \beta}\right\|_{L^{2}}<C .
$$

The mapping

$$
\sum_{\substack{|\alpha|=0 \\|\beta|=0}}^{\infty}(-1)^{\alpha} D^{\alpha}\left(1+|x|^{2}\right)^{\beta / 2}
$$

maps $W^{\prime}$ onto $\mathscr{S}^{\prime\left\{M_{p}\right\}}$ and

$$
B \subset\left(\sum_{\substack{|\alpha|=0 \\|\beta|=0}}^{\infty}(-1)^{\alpha} D^{\alpha}(1+|x|)^{\beta / 2}\right) \widetilde{B},
$$

which implies the assertion.

(ii) From Lemma 1 it follows that $\varphi \in C^{\infty}\left(\mathbf{R}^{n}\right)$ belongs to $\mathscr{S}\left\{M_{p}\right\}$ if and only if $\gamma_{r_{i}, s_{j}}(\phi)<\infty$ for every $r_{i}, s_{j} \in \mathfrak{R}$.

Every norm $\gamma_{r_{i}, s_{j}}, r_{i}, s_{j} \in \mathfrak{R}$, is continuous on $\mathscr{S}_{h}^{M_{p}}, h>0$, so it is continuous on $\mathscr{S}\left\{M_{p}\right\}$.

Since $\mathscr{S}\left\{M_{p}\right\}$ is reflexive, every continuous seminorm $p$ is bounded by the seminorm $p^{B}$, where $B$ is bounded in $\mathscr{S}^{\prime}\left\{M_{p}\right\}$, defined by

$$
p^{B}(\varphi)=\sup \{|\langle f, \varphi\rangle| ; f \in B\} \text {. }
$$


From it follows

$$
p^{B}(\varphi) \leq \sup _{f \in B} \sum_{\substack{|\alpha|=0 \\|\beta|=0}}\left\|\left(1+|x|^{2}\right)^{\beta / 2} D^{\alpha} \varphi\right\|_{L^{2}}\left\|f_{\alpha, \beta}\right\|_{L^{2}},
$$

and by Lemma 1 it follows that there exist $r_{i}$ and $s_{j}$ from $\mathfrak{R}$ such that for some $C>0$

$$
p^{B}(\varphi) \leq C \gamma_{r_{i}, s_{j}}(\phi)
$$

The proof is completed.

Up to the end of the paper we shall assume that conditions (M.1), (M.2), and (M.3) hold.

The following assertion of Komatsu will be used. Note that the first part of this assertion is also proved in [1].

Lemma 5 [6]. Let $K$ be a compact neighbourhood of zero, $r>0$, and $r_{p} \in \mathfrak{R}$.

(i) There is $u \in \mathscr{D}_{K, r / 2}^{\left(M_{p}\right)}$ and $\psi \in \mathscr{D}_{K}^{\left(M_{p}\right)}$ such that

$$
P_{r}(D) u=\delta+\psi
$$

where $P_{r}$ is of form (1).

(ii) There are $u \in C^{\infty}$ and $\psi \in \mathscr{D}_{K}^{\left\{M_{p}\right\}}$ such that

$$
P_{r_{p}}(D) u=\delta+\psi
$$

$$
\operatorname{supp} u \subset K, \quad \sup _{x \in K}\left\{\frac{\mid \partial^{\alpha} u(x)}{R_{|\alpha|} M_{|\alpha|}}\right\} \rightarrow 0, \quad|\alpha| \rightarrow \infty,
$$

where $P_{r_{p}}$ is of the form (3).

Theorem 1. Let $A \subset \mathscr{D}^{\prime *}$.

(i) $A$ is a bounded subset of $\mathscr{D}_{L^{t}}^{\prime\left\{M_{p}\right\}}, t \in[1, \infty]$, if and only if there are $r>0$ and bounded sets $A_{1}$ and $A_{2}$ in $L^{t}$ such that every $f \in A$ is of the form

$$
f=P_{r}(D) F_{1}+F_{2}, \quad F_{1} \in A_{1}, F_{2} \in A_{2} .
$$

(ii) $A$ is an equicontinuous subset of $\widetilde{\mathscr{D}}_{L^{t}}^{\left\{M_{p}\right\}}, t \in[1, \infty]$, if and only if there are $r_{p} \in \mathfrak{R}$ and bounded sets $A_{1}$ and $A_{2}$ in $L^{t}$ such that every $f \in A$ is of the form

$$
f=P_{r_{p}}(D) F_{1}+F_{2}, \quad F_{1} \in A_{1}, F_{2} \in A_{2} .
$$

Proof. Note that we do not know whether the basic space is quasi-barrelled, and because of that we assume in (ii) that $A$ is equicontinuous. We shall prove only assertion (ii) because it is rather complicated. Note that (i) is proved in [1] for $A=\{f\}$.

Since $P_{r_{p}}$ maps $\mathscr{D}_{L^{s}}^{\left\{M_{p}\right\}}, s \in[1, \infty)$, and $\dot{\mathscr{B}}\left\{M_{p}\right\}$ continuously into the same spaces, (11) implies that $A$ is bounded in $\mathscr{D}_{L^{t}}^{\prime\left\{M_{p}\right\}}$.

We shall prove the converse assertion for

$$
t=s /(s-1), \quad s \geq 1 .
$$

For $t=1 \quad(s=\infty)$ the proof is similar. 
Let $\Omega$ be a bounded open set in $\mathbf{R}^{n}$ which contains zero, $K=\bar{\Omega}$, and $\phi \in \mathscr{D}_{K}^{\left\{M_{p}\right\}}$. First we show that, for every $f \in A, f * \phi$ is a continuous linear functional on $\mathscr{D}\left\{M_{p}\right\}$ endowed with the topology of $L^{s}$. Since $A$ is equicontinuous, there are $C>0$ which do not depend on $f \in A$ and $r_{p} \in \mathfrak{R}$ such that for every $\psi \in \mathscr{D}\left\{M_{p}\right\}$

$$
\begin{aligned}
|\langle f * \phi, \psi\rangle| & =|\langle f, \check{\phi} * \psi\rangle| \leq C\|\check{\phi} * \psi\|_{L^{s}, r_{p}} \\
& \leq C\|\phi\|_{K, r_{p}}\|\psi\|_{L^{s}} \leq C_{1}\|\psi\|_{L^{s}} \quad(\check{\phi}(-x)=\phi(x)) .
\end{aligned}
$$

Because $\mathscr{D}\left\{M_{p}\right\}$ is dense in $L^{s}$, it follows that $\{f * \phi ; f \in A\}$ is a set of (continuous) functions bounded in $L^{t}$. Moreover, (12) implies

$$
\sup \left\{\|f * \phi\|_{L^{t}} ; f \in A\right\} \leq C\|\phi\|_{K, r_{p}} .
$$

So if $B$ is a bounded set of $\mathscr{D}_{K}^{\left\{M_{p}\right\}}$, then

$$
\sup \left\{\|f * \phi\|_{L^{i}} ; \phi \in B, f \in A\right\}<\infty .
$$

Next, we show that there is (another) $r_{p} \in \mathfrak{R}$ such that for every $\theta \in \mathscr{D}_{\Omega, r_{p}}^{\left\{M_{p}\right\}}$, $\{f * \theta ; f \in A\}$ is a bounded set in $L^{t}$.

Let $B_{1}$ be the unit ball in $L^{s}$ and $B$ be a bounded subset of $\mathscr{D}_{K}^{\left\{M_{p}\right\}}$. Then for every $f \in A, \psi \in B_{1} \cap \mathscr{D}\left\{M_{p}\right\}$, and $\phi \in B$,

$$
|\langle f * \check{\psi}, \check{\phi}\rangle|=|\langle f * \phi, \psi\rangle| \leq\|f * \phi\|_{L^{\prime}}\|\psi\|_{L^{s}}=\|f * \phi\|_{L^{\prime}} \leq D<\infty,
$$

where $D$ does not depend on $\phi$ and $f$. This implies that $\{f * \check{\psi} ; f \in A, \psi \in$ $\left.B_{1} \cap \mathscr{D}^{\left\{M_{p}\right\}}\right\}$ is bounded in $\mathscr{D}_{K}^{\prime\left\{M_{p}\right\}}$. Since $\mathscr{D}_{K}^{\left\{M_{p}\right\}}$ is barrelled, this family is equicontinuous in $\mathscr{D}_{K}^{\prime\left\{M_{p}\right\}}$. This implies that there exists a neighbourhood of zero in $\mathscr{D}_{K}^{\left\{M_{p}\right\}}$

$$
V_{r_{p}}(\varepsilon)=\left\{\theta \in \mathscr{D}_{K}^{\left\{M_{p}\right\}} ;\|\theta\|_{K, r_{p}} \leq \varepsilon\right\}, \quad \varepsilon>0,
$$

such that

$$
\theta \in V_{r_{p}}(\varepsilon) \Rightarrow|\langle f * \check{\psi}, \check{\theta}\rangle|=|\langle f * \theta, \psi\rangle| \leq 1, \quad \psi \in B_{1} \cap \mathscr{D}^{\left\{M_{p}\right\}}, f \in A .
$$

The same inequality holds for the closure of $V_{r_{p}}(\varepsilon)$ in $\mathscr{D}_{K, r_{p}}^{\left\{M_{p}\right\}}$.

Let $\delta_{k}(t)=k \omega(k t), t \in \mathbf{R}, k \in \mathbf{N}$, where $\omega \in \mathscr{D}^{\left\{M_{p}\right\}}, 0 \leq \omega \leq 1$, $\int_{\mathbf{R}} \omega(t) d t=1$, and let $\delta_{k}(x)=\delta_{k}\left(x_{1}\right), \ldots, \delta_{k}\left(x_{n}\right), x \in \mathbf{R}^{n}, k \in \mathbf{N}$. One can easily prove that for given $\mu \in \mathscr{D}_{\Omega, r_{p}}^{\left\{M_{p}\right\}}, \mu * \delta_{k}, k \in \mathbf{N}, k>k_{0}$, is a sequence from $\mathscr{D}_{K}^{\left\{M_{p}\right\}}$ which converges to $\mu$ in the norm \|\|$_{K, r_{p}}^{\left\{M_{p}\right\}}$. If $\theta \in \mathscr{D}_{\Omega, r_{p}}^{\left\{M_{p}\right\}}$, then, for some $N>0,\|\theta / N\|_{K, r_{p}}^{\left\{M_{p}\right\}}<\varepsilon$ and there is a sequence from $\frac{\Omega, r_{p}}{V_{r_{p}}(\varepsilon)}$ which converges to $\theta / N$ in the norm \|\|$_{K, r_{p}}^{\left\{M_{p}\right\}}$.

This implies that for every $\theta \in \mathscr{D}_{\Omega, r_{p}}^{\left\{M_{p}\right\}}$ there is $C>0$ such that

$$
|\langle f * \check{\psi}, \check{\theta}\rangle|=|\langle f * \theta, \psi\rangle| \leq C, \quad \psi \in B_{1} \cap \mathscr{D}^{\left\{M_{p}\right\}}, f \in A,
$$


and thus,

$$
|\langle f * \theta, \psi\rangle| \leq C\|\psi\|_{L^{s}}, \quad \psi \in \mathscr{D}^{\left\{M_{p}\right\}}, f \in A .
$$

This proves that, for every $\theta \in \mathscr{D}_{\Omega, r_{p}}^{\left\{M_{p}\right\}},\{f * \theta ; f \in A\}$ is a bounded set in $L^{t}$. Lemma 5(ii) implies that for every $f \in A$

$$
f=P_{r_{p}}(u * f)-\psi * f, \quad \text { where } u \in \mathscr{D}_{\Omega, r_{p}}^{\left\{M_{p}\right\}}, \psi \in \mathscr{D}_{\Omega}^{\left\{M_{p}\right\}},
$$

and since $\{u * f ; f \in A\}$ and $\{\psi * f ; f \in A\}$ are bounded sets in $L^{t}$, the proof is completed.

Let $r>0$ (resp. $r_{p} \in \mathfrak{R}$ ) be given. There is $\tilde{r}>0$ (resp. $\tilde{r}_{p} \in \mathfrak{R}$ ) such that for $\varphi \in \mathscr{D}_{K, \tilde{r} / 2}^{\left(M_{p}\right)}$ (resp. $\left.\varphi \in \mathscr{D}_{K, \tilde{r}_{p}}^{\left\{M_{p}\right\}}\right), P_{r} \varphi$ (resp. $\left.P_{r_{p}} \varphi\right)$ is a continuous function. This and the preceding theorem imply the following

Corollary 1. An $f \in \mathscr{D}^{\prime *}$ is from $\mathscr{D}_{L^{t}}^{\left(M_{p}\right)}$ (resp. $\left.\widetilde{\mathscr{D}}^{\prime\left\{M_{p}\right\}}\right), t \in[1, \infty]$, if and only if for every compact set $K$ there is $r>0$ (resp. $r_{p} \in \mathfrak{R}$ ) such that for every $\phi \in \mathscr{D}_{K, r / 2}^{\left(M_{p}\right)}\left(\right.$ resp. $\left.\phi \in \mathscr{D}_{K, r_{p}}^{\left\{M_{p}\right\}}\right) \quad f * \phi \in L^{t}$.

The following is the structural theorem for tempered ultradistributions.

Theorem 2. Let $A \subset \mathscr{D}^{\prime\left(M_{p}\right)}$ (resp. $\left.A \subset \mathscr{D}^{\prime\left\{M_{p}\right\}}\right)$. Then $A$ is a bounded subset of $S^{\prime\left(M_{p}\right)}$ (resp. $A$ is a bounded subset of $S^{\prime\left\{M_{p}\right\}}$ ) if and only if $f$ is of the form

$$
f=P(D) F, \quad F \in A_{1},
$$

where $P$ is an operator of class $\left(M_{p}\right)$ (resp. of class $\left\{M_{p}\right\}$ ) and $A_{1}$ is a set of continuous function on $\mathbf{R}^{n}$ such that for some $k>0$ and some $C>0$ (resp. for every $k>0$ there is $C>0$ ) such that

$$
|F(x)| \leq C \exp (M(k|x|)), \quad F \in A_{1}, \quad x \in \mathbf{R}^{n} .
$$

Proof. We shall prove again the $\left\{M_{p}\right\}$ case since this is rather complicated and the ideas of the proofs for both cases are similar. Clearly (14) implies that by (13) a bounded set in $\mathscr{S}^{\prime}\left\{M_{p}\right\}$ is defined.

Let $A$ be a bounded set in $S^{\prime\left\{M_{p}\right\}}$. For the Fourier transform $\hat{f}(f \in A)$ there are $r_{i}, s_{j} \in \mathfrak{R}$ and $A>0$ which do not depend on $f \in A$ such that

$$
|\langle\hat{f}(\xi), \phi(\xi)\rangle|>A \gamma_{r_{i}, s_{j}}(\phi), \quad \phi \in S^{\left\{M_{p}\right\}} .
$$

For some $D>0$ and $c>0$.

$$
\sup _{\alpha \in N_{0}^{n}}\left\{\frac{\left(1+|x|^{2}\right)^{\alpha / 2}}{M_{|\alpha|}\left(\prod_{i=1}^{|\alpha|} r_{i}\right)}\right\} \leq D \exp (N(c|x|)), \quad x \in \mathbf{R}^{n} .
$$

Let $\tilde{r}_{p}$ and $\rho_{0}$ correspond to $r_{p}, c$ and $C$ in (4) where $C$ is from (6) and $c$ 
from (16). If $\phi \in \mathscr{D}^{\left\{M_{p}\right\}}$, from (4)-(6), it follows that

$$
\begin{aligned}
& \gamma_{r_{i}, s_{j}}\left(\phi / P_{\tilde{r}_{p}}\right) \\
& \leq \sup \left\{\frac{\left\|\left(1+|x|^{2}\right)^{\alpha / 2} \sum_{k \leq \beta}\left(\begin{array}{l}
\beta \\
k
\end{array}\right) \partial^{\beta-k} \phi \partial^{k}\left(1 / P_{\tilde{r}_{p}}\right)\right\|_{L^{2}}}{M_{|\alpha|}\left(\prod_{i=1}^{|\alpha|} r_{i}\right) M_{|\beta-k|}\left(\prod_{j=1}^{|\beta-k|} s_{j}\right) M_{|k|}\left(\prod_{j=1}^{|\alpha|} s_{j}\right)} ; \alpha, \beta \in \mathbf{N}_{0}^{n}\right\} \\
& \leq \sup \left\{\| \sup _{\alpha \in \mathbf{N}_{0}}\left\{\frac{\left(1+|x|^{2}\right)^{\alpha / 2}}{M_{|\alpha|}\left(\prod_{i=1}^{|\alpha|} r_{i}\right)}\right\} 2^{-\beta} \sum_{k \leq \beta}\left(\begin{array}{l}
\beta \\
k
\end{array}\right) \sup _{k}\left\{\frac{\left|\partial^{k}\left(1 / P_{\tilde{r}_{p}}\right)\right|}{M_{|k|}\left(\prod_{j=1}^{|k|}\left(s_{j} / 2\right)\right)}\right\}\right. \\
& \left.\cdot \frac{\left|\partial^{\beta-k} \phi\right|}{M_{|k-\beta|}\left(\prod_{j=1}^{|\beta-k|}\left(s_{j} / 2\right)\right)} \|_{L^{2}} ; k, \beta \in \mathbf{N}_{0}^{n}, k \leq \beta\right\} \\
& \leq D \sup \left\{\| \exp N(c|x|) \sup _{k}\left\{\frac{|k| ! d^{-k}}{M_{|k|}\left(\prod_{j=1}^{|k|}\left(s_{j} / 2\right)\right)}\right\}\right. \\
& \cdot \exp (-N(|x|) / C) 2^{-\beta} \sum_{k \leq \beta}\left(\begin{array}{l}
\beta \\
k
\end{array}\right) \frac{\left|\partial^{\beta-k} \phi\right|}{M_{|\beta-k|}\left(\prod_{j=1}^{|\beta-k|}\left(s_{j} / 2\right)\right)} \|_{L^{2}} ; \\
& \left.k, \beta \in \mathbf{N}_{0}^{n}, k \leq \beta\right\} \\
& \leq C_{1} \sup \left\{2^{-\beta} \sum_{k \leq \beta}\left(\begin{array}{l}
\beta \\
k
\end{array}\right) \frac{\left\|\partial^{\beta-k} \phi\right\|_{L^{2}}}{M_{|\beta-k|}\left(\prod_{j=1}^{|\beta-k|}\left(s_{j} / 2\right)\right)}, k, \beta \in \mathbf{N}_{0}, k \leq \beta\right\} \\
& \leq C_{1}\|\phi\|_{L^{2}, s_{j / 2}} \text {. }
\end{aligned}
$$

Thus, (15) implies that for suitable $C_{1}>0$

$$
\begin{aligned}
\left|\left\langle\hat{f}(\xi) / P_{\tilde{r}_{p}}(\xi), \phi(\xi)\right\rangle\right| & =\left|\left\langle\hat{f}(\xi), \phi(\xi) / P_{\tilde{r}_{p}}(\xi)\right\rangle\right| \\
& \leq C_{1}\|\phi\|_{L^{s}, s_{p / 2}}, \quad f \in A, \phi \in \mathscr{D}_{L^{2}}^{\left\{M_{p}\right\}} .
\end{aligned}
$$

This implies that $\left\{\hat{f} / P_{\tilde{r}_{p}} ; f \in A\right\}$ is equicontinuous in $\widetilde{\mathscr{D}}_{L^{2}}^{\prime\left\{M_{p}\right\}}$, and by Theorem 1 every $\hat{f}(f \in A)$ is of the form

$$
\hat{f}(\xi)=P_{\tilde{r}_{p}}(\xi)\left(P_{\tilde{r}_{p}}(D) \widetilde{F}_{1}(\xi)+\widetilde{F}_{2}(\xi)\right), \quad \widetilde{F}_{1} \in \widetilde{A}_{1}, \widetilde{F}_{2} \in \widetilde{A}_{2},
$$

where $\tilde{A_{1}}$ and $\tilde{A_{2}}$ are bounded subsets of $L^{2}$. By the inverse Fourier transform we obtain

$$
f(x)=P_{\tilde{r}_{p}}(D)\left(P_{\tilde{r}_{p}}(x) F_{1}(x)+F_{2}(x)\right), \quad F_{1} \in A_{1}, \quad F_{2} \in A_{2},
$$

where $A_{1}$ and $A_{2}$ are bounded subsets of $L^{2}$. Put

$$
\begin{gathered}
F(x)=\int_{0}^{x_{1}} \cdots \int_{0}^{x_{n}}\left(P_{\tilde{r_{p}}}(t) F_{1}(t)+F_{2}(t)\right) d t_{1} \cdots d t_{n}, \\
\quad x \in \mathbf{R}^{n}, \quad F_{1} \in A_{1}, \quad F_{2} \in A_{2}, \\
P(D)=P_{\tilde{r}_{p}}(D) \frac{\partial^{n}}{\partial x_{1} \cdots \partial x_{n}} .
\end{gathered}
$$


From (4) it follows that

$$
\begin{aligned}
|F(x)| & \leq C \exp (\tilde{\tilde{N}}(|x|))\left(1+|x|^{2}\right)^{n} \int_{0}^{x} \frac{F_{1}(t)+F_{2}(t)}{\left(1+|t|^{2}\right)^{n}} d t \\
& \leq C_{1}\left(\left\|F_{1}\right\|_{L^{2}}+\left\|F_{2}\right\|_{L^{2}}\right)\left(1+|x|^{2}\right)^{n} \exp \tilde{\tilde{N}}(x), \quad x \in \mathbf{R}^{n} .
\end{aligned}
$$

Since for every $k>0$ there is $\rho_{k}>0$ such that [4]

$$
\tilde{\tilde{N}}(x) \leq M(k|x|), \quad|x|>\rho_{k},
$$

(14) follows and the theorem is proved.

\section{BOUNDED SETS IN $\mathscr{D}^{\prime *}$}

In this section we shall give two theorems analogous to the corresponding theorems for distributions [12].

Lemma 6. Let $f \in \mathscr{D}^{\prime *}, \Omega$ be a bounded open set, and $K$ be a compact set. The mapping

$$
G_{f}: \mathscr{D}_{K}^{*} \times \mathscr{D}_{K}^{*} \rightarrow L^{\infty}(\Omega), \quad(\alpha, \beta) \rightarrow G_{f}(\alpha, \beta)=f * \alpha * \beta_{\mid \Omega},
$$

is continuous. (Symbol $\left.\right|_{\mid \Omega}$ means the restriction on $\Omega$.)

Proof. Let $K_{1}=K+K=\{x+y ; x, y \in K\}$ and $\Omega_{1}$ be an open bounded set such that $\Omega_{1} \supset \overline{\Omega-K_{1}}$. From [4, Theorem 10.3] it follows that

$$
f_{\mid \Omega_{1}}=P(D) F,
$$

where $F$ is continuous on $\overline{\Omega_{1}}$ and $P=P_{r}$ is of form (1) (resp. $P=P_{r_{p}}$ is of form (3)). Let $\varepsilon>0$ be given. There are $\tilde{r}>0$ (resp. $\tilde{r}_{p} \in \mathfrak{R}$ ) and $\delta>0$ such that

$$
\begin{gathered}
\alpha \in V_{\tilde{r}}(\delta)=\left\{\phi \in \mathscr{D}_{K}^{\left(M_{p}\right)} ;\|\phi\|_{K, \tilde{r}}<\delta\right\} \Rightarrow \sup _{x \in K}\left\{\left|P_{r}(D) \alpha(x)\right|\right\}<\varepsilon \\
\left(\alpha \in V_{\tilde{r}_{p}}(\delta)=\left\{\phi \in \mathscr{D}_{K}^{\left\{M_{p}\right\}} ;\|\phi\|_{K, \tilde{r}_{p}}>\delta\right\} \Rightarrow \sup _{x \in K}\left\{\left|P_{r_{p}}(D) \alpha(x)\right|\right\}<\varepsilon\right) .
\end{gathered}
$$

Let $\alpha, \beta \in V_{\tilde{r}}(\delta)$ (resp. $\left.\alpha, \beta \in V_{\tilde{r}_{p}}(\delta)\right)$ and $x \in \Omega$. In the $\left(M_{p}\right)$-case

$$
\begin{aligned}
|(f * \alpha * \beta)(x)| & \leq\left|\left\langle P_{r}(D) F(t),(\alpha * \beta)(x-t)\right\rangle\right| \\
& =\left|\left\langle F(t),\left(P_{r} \alpha * \beta\right)(x-t)\right\rangle\right| \\
& \leq \int_{\Omega_{1}}\left|F(t)\left(P_{r} \alpha * \beta\right)(x-t)\right| d t \\
& \leq C \sup _{t \in \Omega_{1}}\left\{\left|\left(P_{r}(D) \alpha * \beta\right)(x-t)\right|\right\} \\
& \leq C_{1} \sup _{t \in K}\left\{\left|P_{r}(D) \alpha(t)\right|\right\} \sup _{t \in K}\{|\beta(t)|\} \leq C_{1} \varepsilon^{2},
\end{aligned}
$$

where $C=\int_{\Omega} F(t) d t, C_{1}=C \cdot \operatorname{mes} \Omega$.

The same holds in the $\left\{M_{p}\right\}$ case. Thus, in both cases we obtain the continuity of $G_{f}$. 
Theorem 3. Let $B^{\prime}$ be a subset of $\mathscr{D}^{\prime *}$.

(i) $B^{\prime}$ is bounded in $\mathscr{D}^{\prime *}$ if and only if for every bounded open set $\Omega \subset \mathbf{R}^{n}$ and every $\phi \in \mathscr{D}^{*}$

$$
\sup \left\{|(T * \phi)(x)| ; x \in \Omega, T \in B^{\prime}\right\}<\infty .
$$

(ii) $B^{\prime}$ is bounded in $\mathscr{D}^{\prime *}$ if and only if for every open bounded set $\Omega \subset \mathbf{R}^{n}$ and every open bounded neighbourhood of zero $\omega$ there is $r>0$ (resp. there is $r_{p} \in \mathfrak{R}$ ) such that for every $\phi \in \mathscr{D}_{\omega, r / 2}^{M_{p}}$ (resp. for every $\phi \in \mathscr{D}_{\omega, r_{p}}^{\left\{M_{p}\right\}}$ ) (17) holds. Proof. (i) Let $B^{\prime}$ be a bounded set in $\mathscr{D}^{* *}$. Let $\phi \in \mathscr{D}^{*}$, supp $\phi=K$, and $\Omega_{1}$ and $\Omega_{2}$ be open bounded sets in $\mathbf{R}^{n}$ such that $\Omega_{1} \supset \overline{\Omega-K}, \Omega_{2} \supset \overline{\Omega_{1}}$. The set $B_{\Omega_{2}}^{\prime}$ of restrictions $T_{\mid \Omega_{2}}, T \in B^{\prime}$, is bounded in $\mathscr{D}^{\prime *}\left(\Omega_{2}\right)$.

Consider the set $B=\left\{\phi_{x} ; x \in \Omega\right\}$, where

$$
\phi_{x}: t \rightarrow \phi(x-t), \quad t \in \Omega_{2}, x \in \Omega .
$$

All the elements of $B$ have the supports contained in $\overline{\Omega_{1}}$. Moreover, $B$ is bounded in $\mathscr{D}^{*}\left(\Omega_{2}\right)$. Because of that, for some $C>0$,

$$
\left|\left\langle T, \phi_{x}\right\rangle\right|=|(T * \phi)(x)|<C, \quad T \in B^{\prime}, x \in \Omega .
$$

Now, assume that, for every $\phi \in \mathscr{D}^{\prime *},(17)$ holds. As in Lemma 6, for every $T \in B^{\prime}$ we denote by $G_{T}$ the continuous linear mapping from $\mathscr{D}_{K}^{*} \times \mathscr{D}_{K}^{*}$ into $L^{\infty}(\Omega)$. The set $\mathscr{G}=\left\{G_{T} ; T \in B^{\prime}\right\}$ is pointwisely bounded. Namely, (17) implies that for fixed $\alpha \in \mathscr{D}_{K}^{*}$ the set $\left\{T * \alpha ; T \in B^{\prime}\right\}$ is bounded in $\mathscr{D}_{K}^{\prime *}$, and thus, for fixed $\beta \in \mathscr{D}_{K}^{*}$, there exist $C_{\beta}>0$ such that

$$
\left|G_{T}(\alpha, \beta)(x)\right|=|((T * \alpha) * \beta)(x)| \leq C_{\beta}, \quad G_{T} \in \mathscr{G}, x \in \Omega .
$$

Since $\mathscr{D}_{K}^{*} \times \mathscr{D}_{K}^{*}$ is barrelled, the family $\mathscr{G}$ is equicontinuous. This implies that there exists a neighbourhood of zero $V_{r}(\varepsilon)$ (resp. $V_{r_{p}}(\varepsilon)$ ) in $\mathscr{D}_{K}^{\left(M_{p}\right)}$ (resp. in $\left.\mathscr{D}_{K}^{\left\{M_{p}\right\}}\right)$ such that

$$
\begin{gathered}
\alpha, \beta \in V_{r}(\varepsilon) \Rightarrow \sup \left\{|(T * \alpha * \beta)(x)| ; \quad T \in B^{\prime}, \quad x \in \Omega\right\} \leq 1 \\
\text { (resp. } \left.\alpha, \beta \in V_{r_{p}}(\varepsilon) \Rightarrow \sup \left\{|(T * \alpha * \beta)(x)| ; \quad T \in B^{\prime}, \quad x \in \Omega\right\} \leq 1\right) .
\end{gathered}
$$

The same holds for $\alpha, \beta$ being in the closure $\overline{V_{r}(\varepsilon)}$ (resp. $\overline{V_{r_{p}}(\varepsilon)}$ ) in $\mathscr{D}_{K, r / 2}^{M_{p}}$ (resp. $\mathscr{D}_{K, r_{p}}^{\left\{M_{p}\right\}}$.

This implies that for arbitrary $\theta, \mu \in \mathscr{D}_{\omega, r / 2}^{\left(M_{p}\right)}$ (resp. $\theta, \mu \in \mathscr{D}_{\omega, r_{p}}^{\left\{M_{p}\right\}}$ )

$$
\sup \left\{|(T * \theta * \mu)(x)| ; T \in B^{\prime}, x \in \Omega\right\}<\infty .
$$

By using two times (9) (resp. (10)) we have that on $\Omega$

$$
T=P_{r}(D) P_{r}(D)(T * u * u)-P_{r}(D)(T * u * \psi)-T * \psi, \quad T \in B^{\prime}
$$

(resp. $\left.T=P_{r_{p}}(D) P_{r_{p}}(D)(T * u * u)-P_{r_{p}}(D)(T * u * \psi)-T * \psi, T \in B^{\prime}\right)$. Because $u \in \mathscr{D}_{\omega, r / 2}\left\{M_{p}\right\}$ and $\psi \in \mathscr{D}_{\omega}^{\left(M_{p}\right)}$ (resp. $u \in \mathscr{D}_{\omega, r_{p}}^{\left\{M_{p}\right\}}$ and $\psi \in \mathscr{D}_{K}^{\left\{M_{p}\right\}}$ ), sets $\left\{T * u * u ; T \in B^{\prime}\right\},\left\{T * u * \psi ; T \in B^{\prime}\right\}$, and $\left\{T * \psi ; t \in B^{\prime}\right\}$ are bounded in $L^{\infty}(\Omega)$. This implies that $\left.\sup \{\mid\langle T, \phi\rangle\} \mid ; T \in B^{\prime}\right\}<\infty$ for every $\phi \in \mathscr{D}^{*}$ with supp $\phi \subset \Omega$. Since $\Omega$ is an arbitrary open bounded set in $\mathbf{R}^{n}$, it follows that $B^{\prime}$ is bounded in $\mathscr{D}^{\prime *}$. 
(ii) We only have to prove that for $B^{\prime}$ being bounded in $\mathscr{D}^{\prime *}$ the assertion in (ii) holds. By [4, Theorem 10.3] there is a set $B$ of continuous functions uniformly bounded on $\overline{\Omega+\omega}$ and $P_{r}$ (resp. $P_{r_{p}}$ ) such that every $T \in B^{\prime}$ is of the form $T=P_{r} F_{\mid(\Omega+\omega)}$ (resp. $\grave{T}=P_{r_{p}} F_{\mid(\Omega+\omega)}$ ) for some $F \in B$. There exists $\tilde{r}$ (resp. $\tilde{r}_{p} \in \mathfrak{R}$ ) such that $P_{r}(D) \phi$ is continuous if $\phi \in \mathscr{D}_{\omega, \tilde{r} / 2}^{\left\{M_{p}\right\}}$ (resp. $P_{r_{p}}(D) \phi$ is continuous if $\phi \in \mathscr{D}_{\omega, \tilde{r} / p}^{\left\{M_{p}\right\}}$.

Since, for $T \in B^{\prime}, \phi \in \mathscr{D}_{\omega, \tilde{r} / 2}^{M_{p}}$ (resp. $\left.\phi \in \mathscr{D}_{\omega, \tilde{r}_{p}}^{\left\{M_{p}\right\}}\right), T * \phi=F * P_{r}(D) \phi$ (resp. $\left.T * \phi=F * P_{\tau_{p}}(D) \phi\right), x \in \Omega$, the assertion follows.

Theorem 4. Let $f_{\nu}$ be a sequence in $\mathscr{D}^{\prime *}$ : Then:

(i) $f_{\nu}$ converges to 0 in $\mathscr{D}^{\prime *}$ if and only if, for every $\phi \in \mathscr{D}^{*}$ and every open bounded set $\Omega \in \mathbf{R}, f_{\nu} * \phi$ converges to 0 uniformly on $\Omega$.

(ii) $f_{\nu}$ converges to 0 in $\mathscr{D}^{\prime *}$ if and only if for every open and bounded set $\Omega \subset \mathbf{R}^{n}$ and every open bounded neighbourhood of zero $\omega$ there is $r>0$ (resp. there is $\left.r_{p} \in \mathfrak{R}\right)$ such that for every $\phi \in \mathscr{D}_{\omega, r / 2}^{M_{p}}$ (resp. $\phi \in \mathscr{D}_{\omega, r_{p}}^{\left\{M_{p}\right\}} f_{\nu} * \phi$ converges to 0 uniformly on $\Omega$.

We omit the proof but only remark that, in order to prove this, one has to use the equicontinuity of the mapping

$$
\mathscr{D}_{K}^{*} \times \mathscr{D}_{K}^{*} \ni \alpha, \beta \rightarrow f_{\nu} * \alpha * \beta \in L^{\infty}(\Omega) \quad(\nu \in \mathbf{N})
$$

and that for every fixed $\alpha, \beta, f_{\nu} * \alpha * \beta$ converges to 0 uniformly on $\Omega$.

\section{REFERENCES}

1. I. Cioranescu, The characterization of the almost-periodic ultradistributions of Beurling type, Proc. Amer. Math. Soc. 116 (1992), 127-134.

2. I. M. Gelfand and G. E. Shilov, Generalized function, Vol. 2, Spaces of Fundamental and Generalized Functions, Academic Press, New York and London, 1968.

3. J. Harvat, Topological vector spaces and distributions, Addison-Wesley, Reading, MA, 1966.

4. H. Komatsu, Ultradistributions, I: Structure theorems and a characterization, J. Fac. Sci. Univ. Tokyo Sect. IA Math. 20 (1973), 23-105.

5. _ Ultradistributions, III: Vector valued ultradistributions and the theory of kernels, J. Fac. Sci. Univ. Tokyo Sect. IA Math. 29 (1982), 653-718.

6. _. Microlocal analysis in Gevrey class and complex domains, UTYO-MATH 91-15 (1991), Lecture delivered at CIME, Inter. Math. Summer Institute: Mycrolocal Analysis and Applications, Montecatini, 1989 (to appear in Lecture Notes).

7. D. Kovačević and S. Pilipović, Structural properties of the space of tempered ultradistributions, Proc. Conf. Complex Analysis and Application '91 with Symposium on Generalized functions (Varna, 1991) (to appear).

8. _ Integral transformations of tempered ultradistributions, preprint.

9. H. J. Patzsche, Generalized functions and the boundary values of holomorphic function, J. Fac. Sci. Univ. Tokyo Sect. IA Math. 31 (1984), 391-431.

10. S. Pilipović, Tempered ultradistributions, Boll. Un. Mat. Ital. B 2 (1988), 235-251.

11. St. Pauli 40 (1991), 15-27.

12. L. Schwartz, Theorie des distributions. I, II, 2nd ed., Hermann, Paris, 1966.

Institute of Mathematics, University of Novi Sad, 21000 Novi Sad, Trg Dositeja Obradovića 4, Yugoslavia

E-mail address: pilipovic\%unsim@yubgef51.bitnet 